\title{
EXPÉRIENCES NUMERIQUES SUR L'ÉJECTION DE MASSES \\ DEPUIS LE CENTRE D'UN DISQUE D'ÉTOILES
}

\author{
S. CLAIREMIDI et A. HAYLI \\ Observatoire de Besançon et Faculté des Sciences, Besançon, France
}

\begin{abstract}
Numerical experiments on simple or symmetric ejections of massive bodies from the nucleus of a disk of stars are presented. The ejected masses range from 0.04 to 0.1 of the total mass. Either spiral features or a barred structure appear but do not last for a long time. Material arms start leading and become trailing.
\end{abstract}

\section{Introduction}

Des preuves s'accumulent depuis quelques années qui accréditent la thèse de l'éjection par le noyau de certaines galaxies de nuages gazeux massifs, de particules relativistes et d'objets massifs cohérents. Nous renvoyons pour la bibliographie à O'Connell (1970).

L'une des hypothèses formulées sur la nature du noyau est qu'il serait composé essentiellement d'un objet supermassif capable de fragmentations récurrentes et dont l'activité régirait le comportement de la galaxie.

Les observations montrent un objet brillant à l'extrémité d'un bras de certaines galaxies (Arp, 1969) ou encore des paires de radio-sources alignées avec des galaxies parentes (Van der Kruit, 1971) qui pourraient avoir été éjectées de la galaxie mère.

Les expériences numériques décrites ci-dessous ont été suggérées par cette hypothèse. Leur objet est l'étude du comportement dynamique qu'aurait un disque d'étoiles si son centre éjectait une fraction de sa masse sous la forme d'un ou deux objets cohérents. On a voulu voir si des éjections d'objets massifs pouvaient être responsables de l'amorce d'une structure spirale dans le disque d'étoiles.

\section{Expériences numériques}

Dans les trois cas présentés ci-dessous on a adopté une représentation du disque d'étoiles analogue à celle de Toomre et Toomre (1972). On a supposé que toute la masse du disque, soit $10^{11} M_{\odot}$ était concentrée en un point. Les étoiles du disque sont des objets tests disposés sur des cercles de rayons 5, 7.5, 10, 12.5 et $15 \mathrm{kpc}$. Ils ont une vitesse circulaire.

Pour éviter les difficultés numériques dans l'intégration des équations du mouvement, on a supposé que la masse éjectée se trouve à $100 \mathrm{pc}$ du centre à l'instant $T=0$. L'éjection se fait toujours dans le plan du disque. L'unité de temps vaut $10^{8}$ ans.

On est réduit à intégrer, pour le mouvement d'une étoile test quelconque un problème de trois corps (dans le cas d'une seule éjection) ou de quatre corps (dans le cas de deux éjections) particulièrement simple. 
2.1. ÉJECTION D'UNE MASSE UNIQUe De $10^{10} M_{\odot}$, SOIT $1 / 10$ DE LA MASSE TOTALE

On prend comme vitesse initiale du corps éjecté $v=2400 \mathrm{~km} \mathrm{~s}^{-1}$. Figure 1. Les étoiles ont été suivies pendant 7 unités de temps correspondant à un peu plus d'une révolution complète pour celles qui sont le plus à l'extérieur du disque, et à 7 révolutions pour les plus intérieures. Au temps $T=7$ la masse éjectée est à environ $100 \mathrm{kpc}$ du centre du disque et sa vitesse est de $150 \mathrm{~km} \mathrm{~s}^{-1}$.

Dès $T=1$ on voit se dessiner l'amorce d'un bras qui devance le disque dans le sens de la rotation. Puis le bras se déforme et finit par traîner.

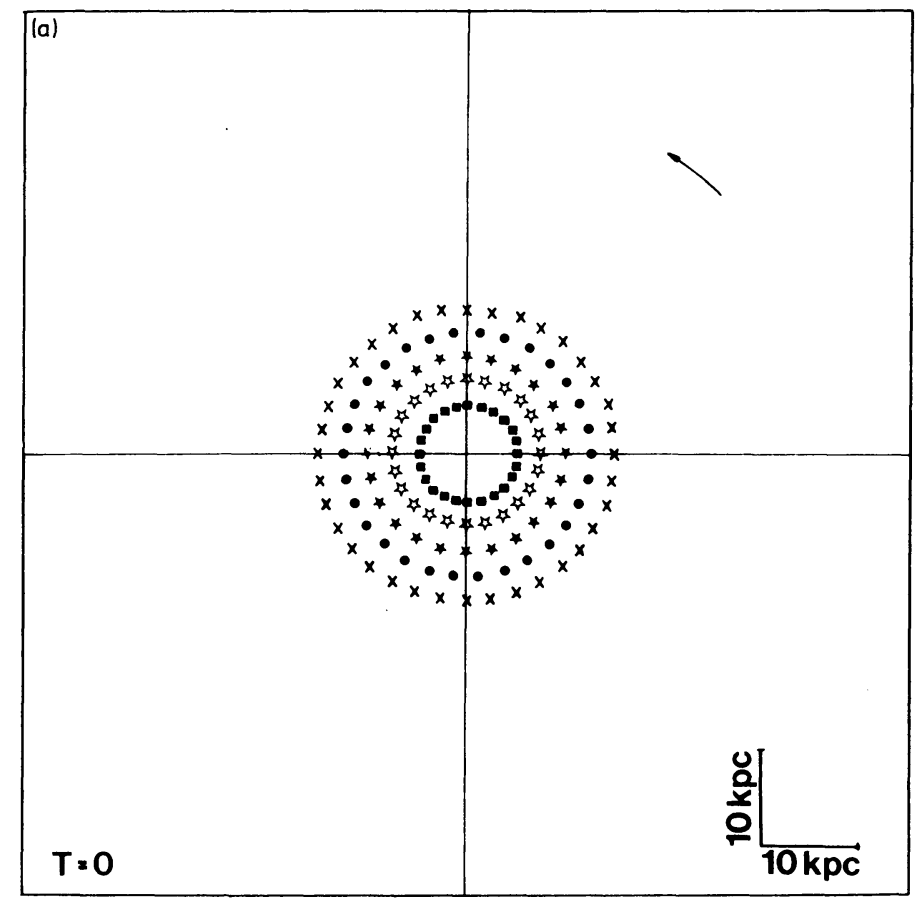

Fig. la. 


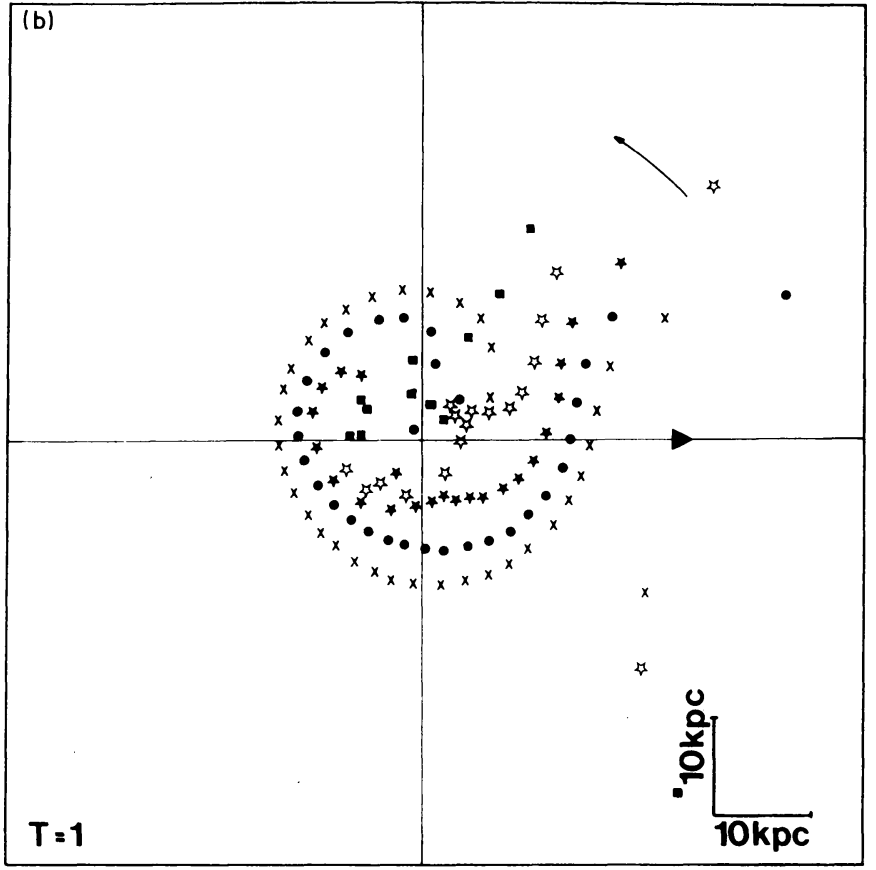

Fig. $1 b$.

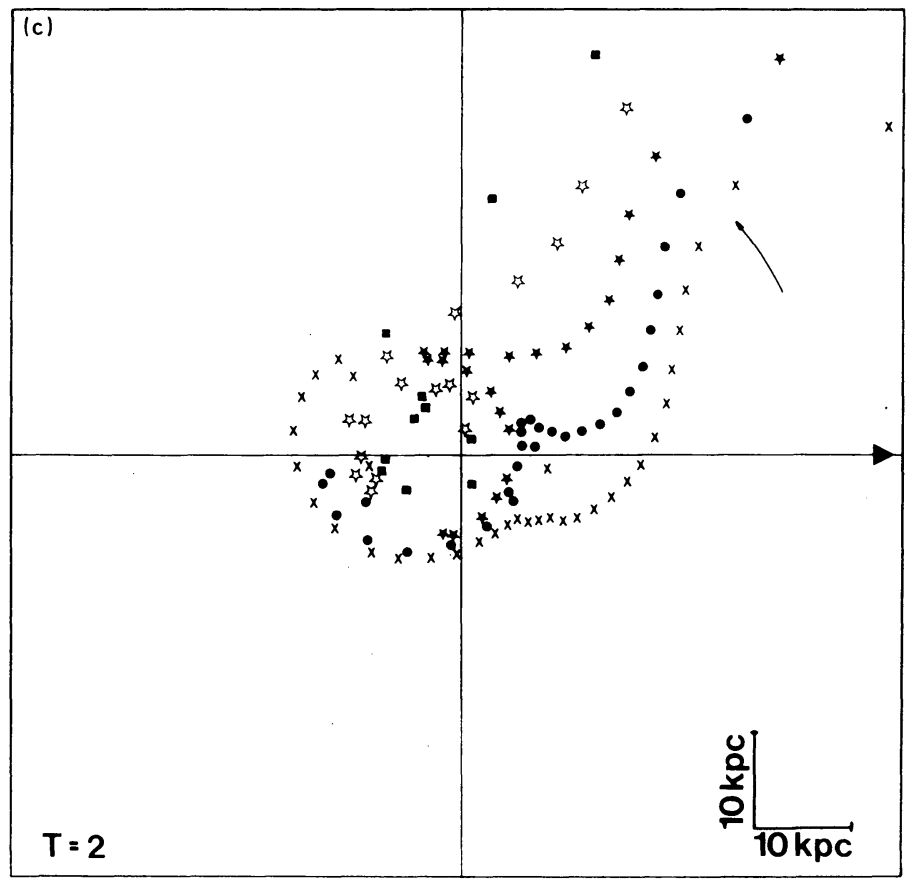

Fig. 1c. 


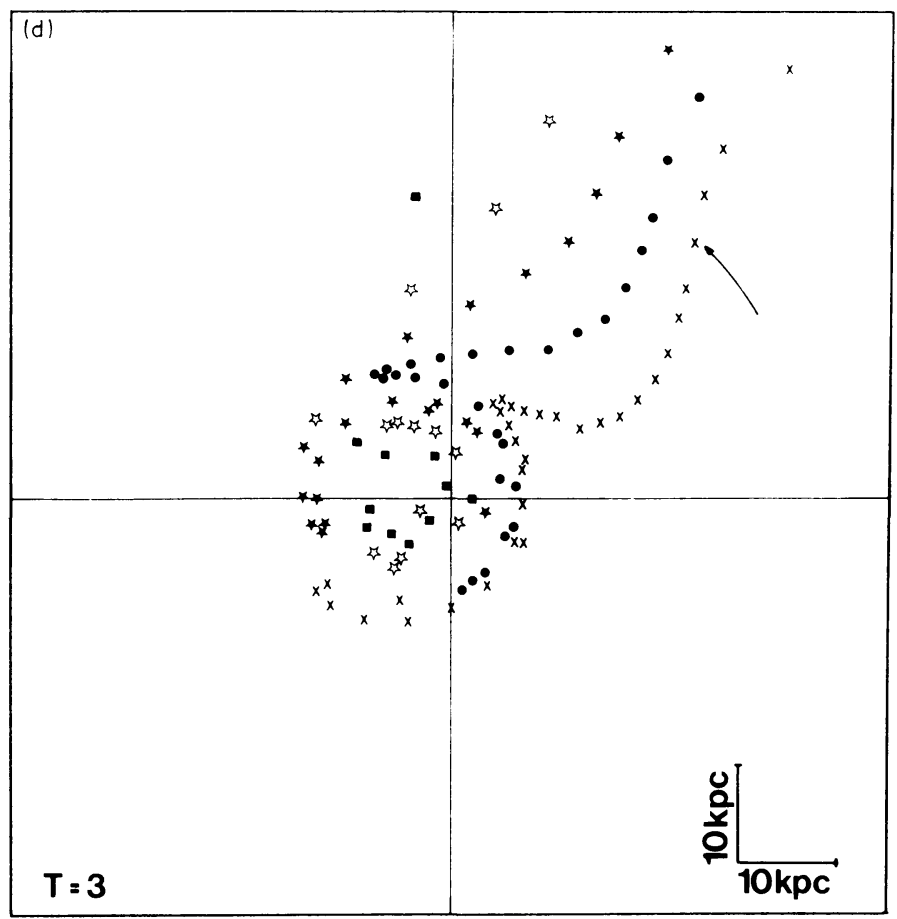

Fig. 1d.

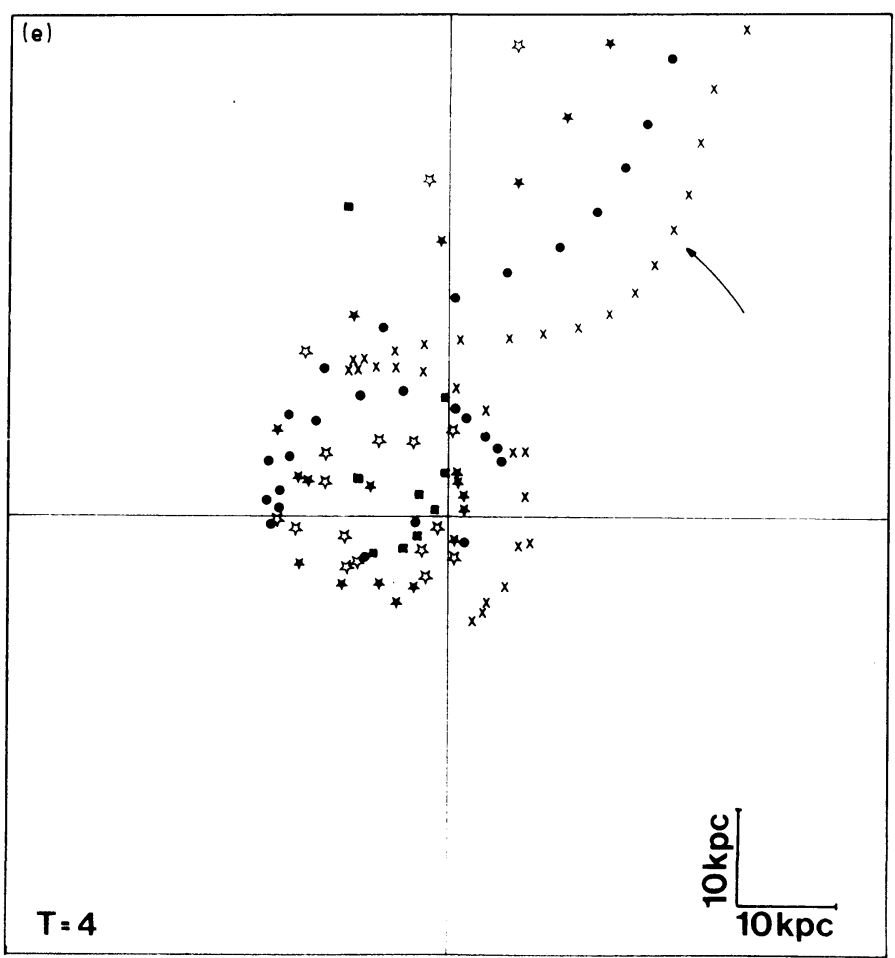

Fig. le. 


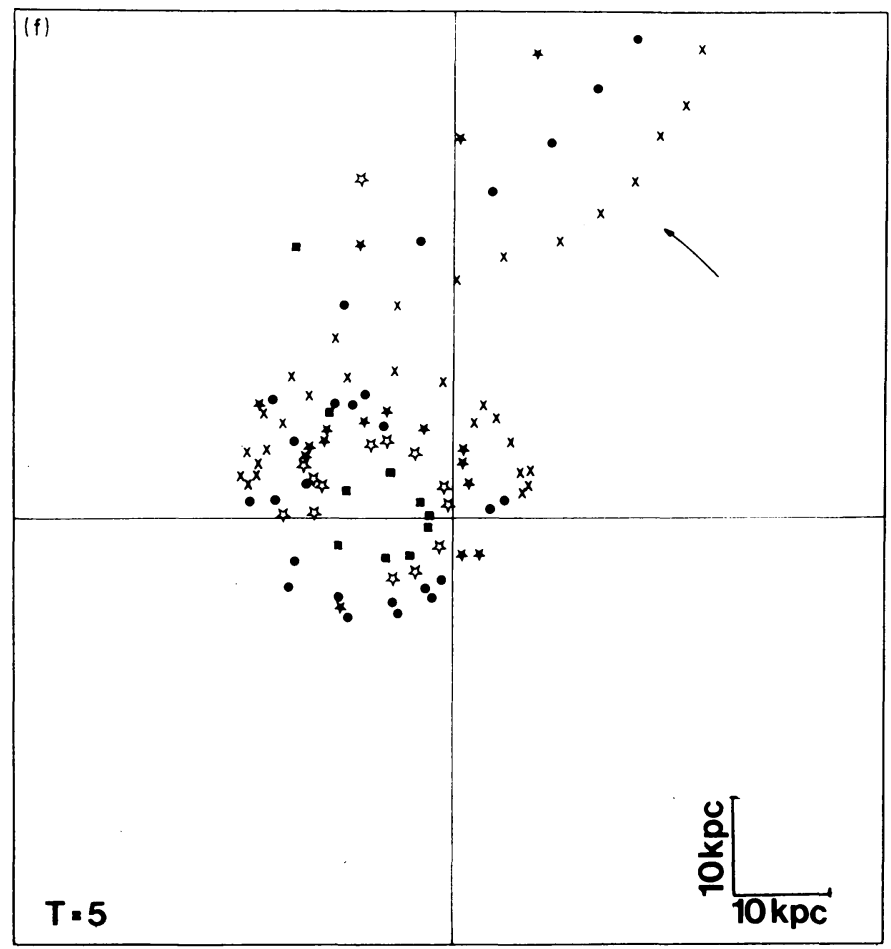

Fig. If.

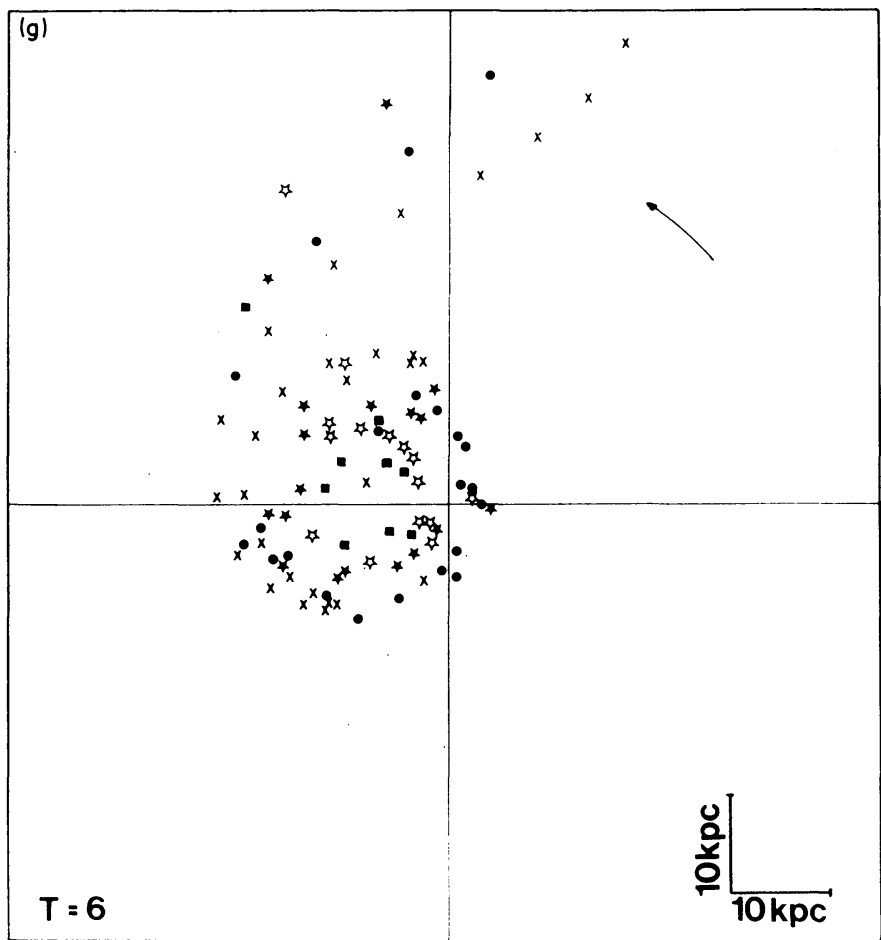

Fig. $1 \mathrm{~g}$. 


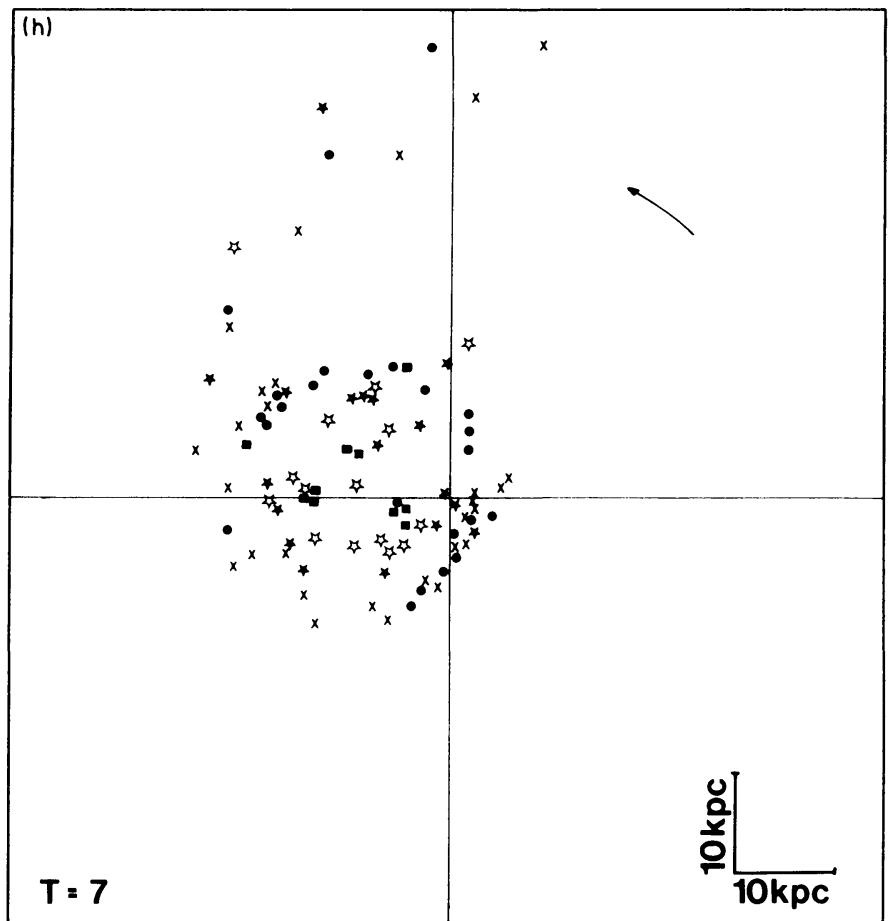

Fig. 1 (a) à (h). Toute la masse du disque, soit $10^{11} M_{\odot}$ est au centre. Les étoiles qui se trouvent à la même distance du centre à l'instant $T=0$ sont représentées par le même symbole. La flèche indique le sens de rotation d'ensemble du disque. Un fragment de $10^{10} M_{\odot}$ est éjecté à $T=0$. Il est représenté par le signe sur les Figures (b) et (c). On a choisi un système de référence lié à la masse centrale. On voit se former un bras qui devient traînant à partir de $T=6$.

2.2. ÉJECTION SYMÉTRIQUE DE DEUX MASSES ÉGALES, CHACUNE, À $0.2 \times 10^{10} M_{\odot}$

Les vitesses initiales sont de $2700 \mathrm{~km} \mathrm{~s}^{-1}$. Figure 2 . Les étoiles ont été suivies pendant 10 unités de temps. La figure 2 ne montre l'évolution du système que jusqu'à $T=7$. A la fin du calcul les masses éjectées sont à $120 \mathrm{kpc}$ de part et d'autre de la masse centrale et leur vitesse est de $120 \mathrm{~km} \mathrm{~s}^{-1}$ environ.

Pendant les 4 premières unités de temps on voit apparaître deux bras qui devancent le disque dans sa rotation, puis les bras disparaissent et la configuration ressemble davantage à celle d'une galaxie spirale barrée. Au delà de $T=8$ la structure du système devient très confuse. 


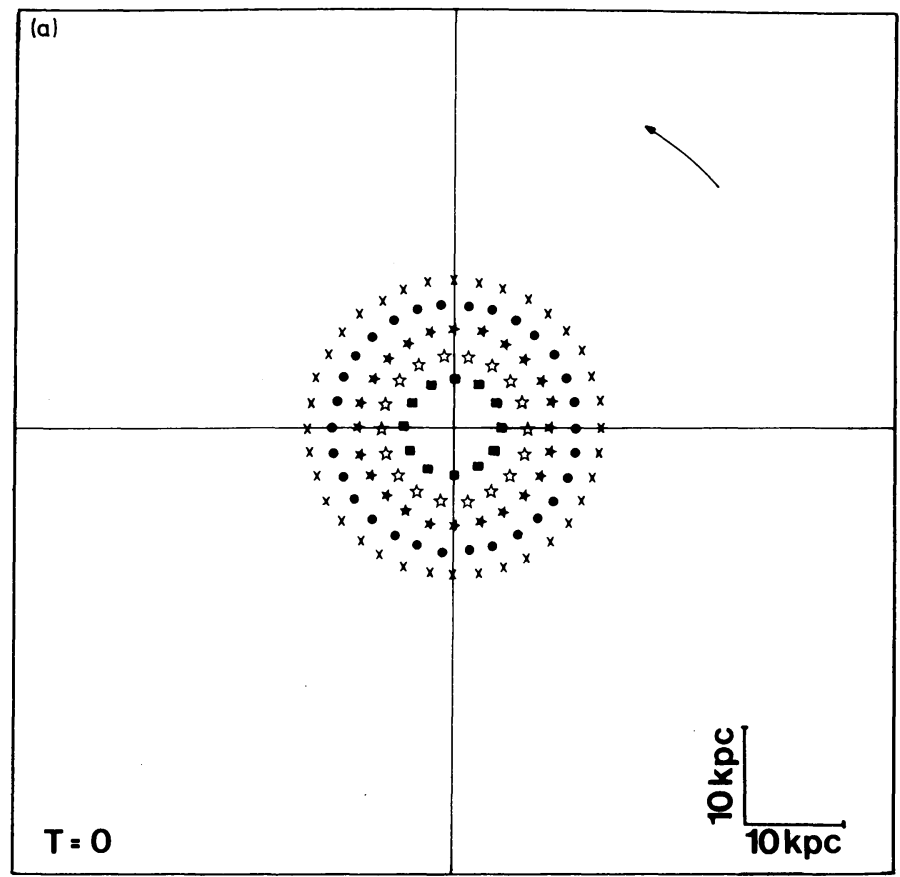

Fig. 2a.

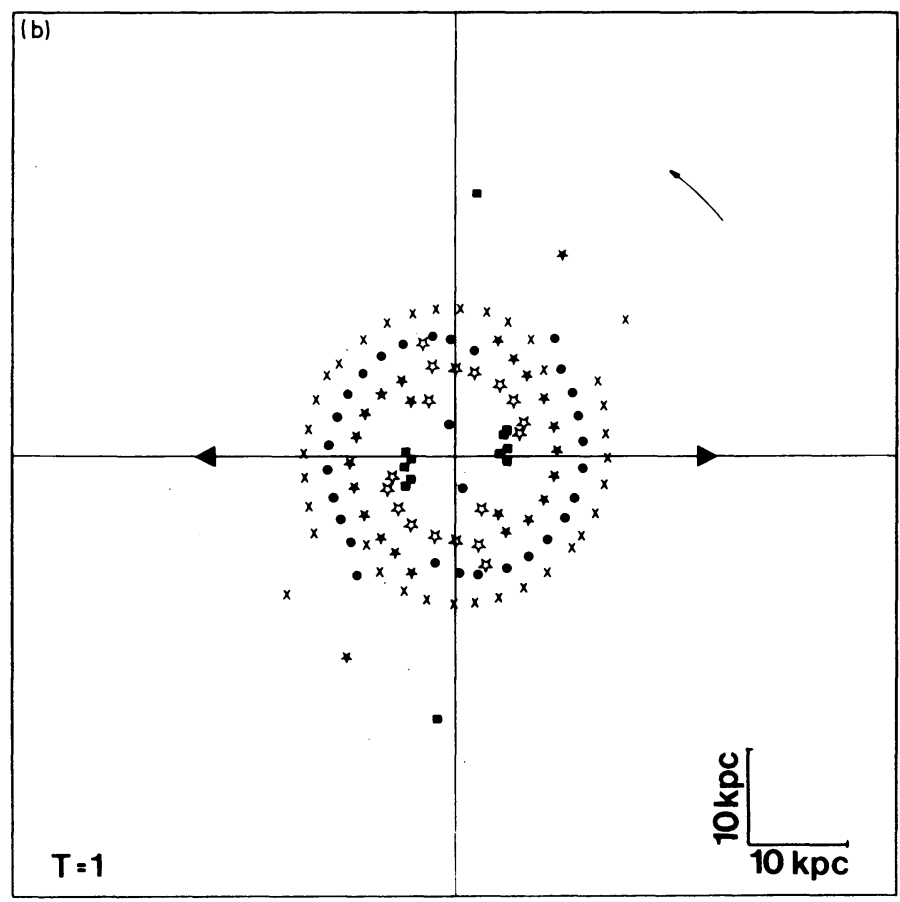

Fig. 2b. 


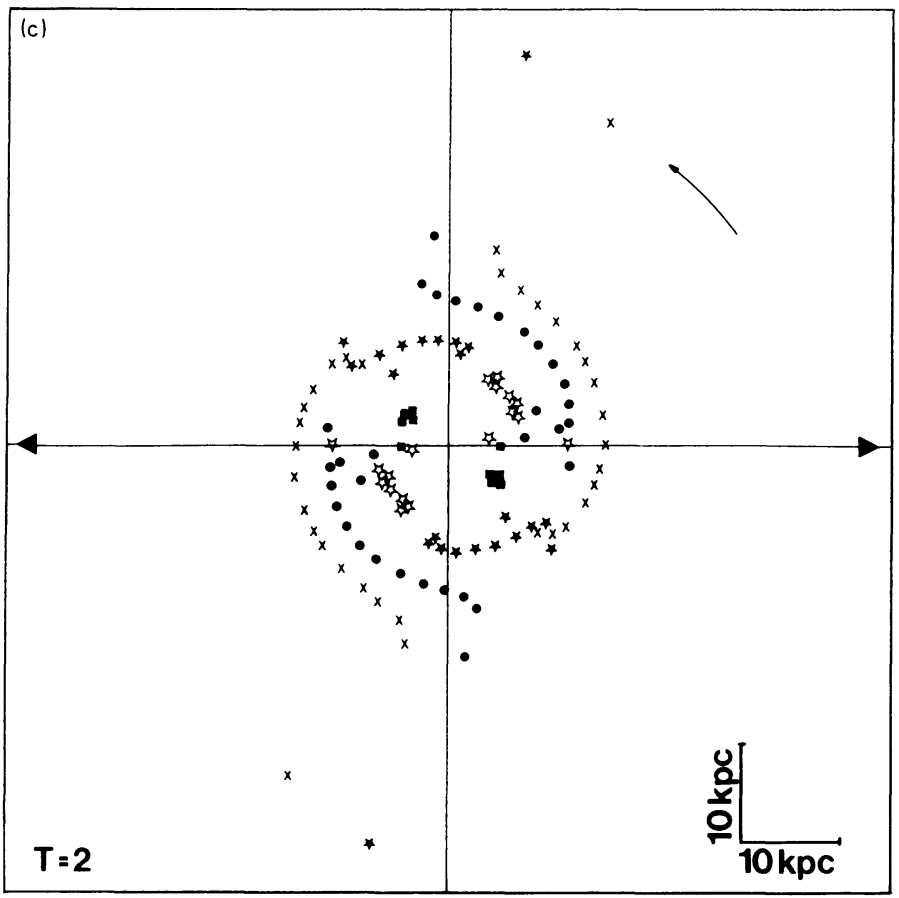

Fig. 2c.

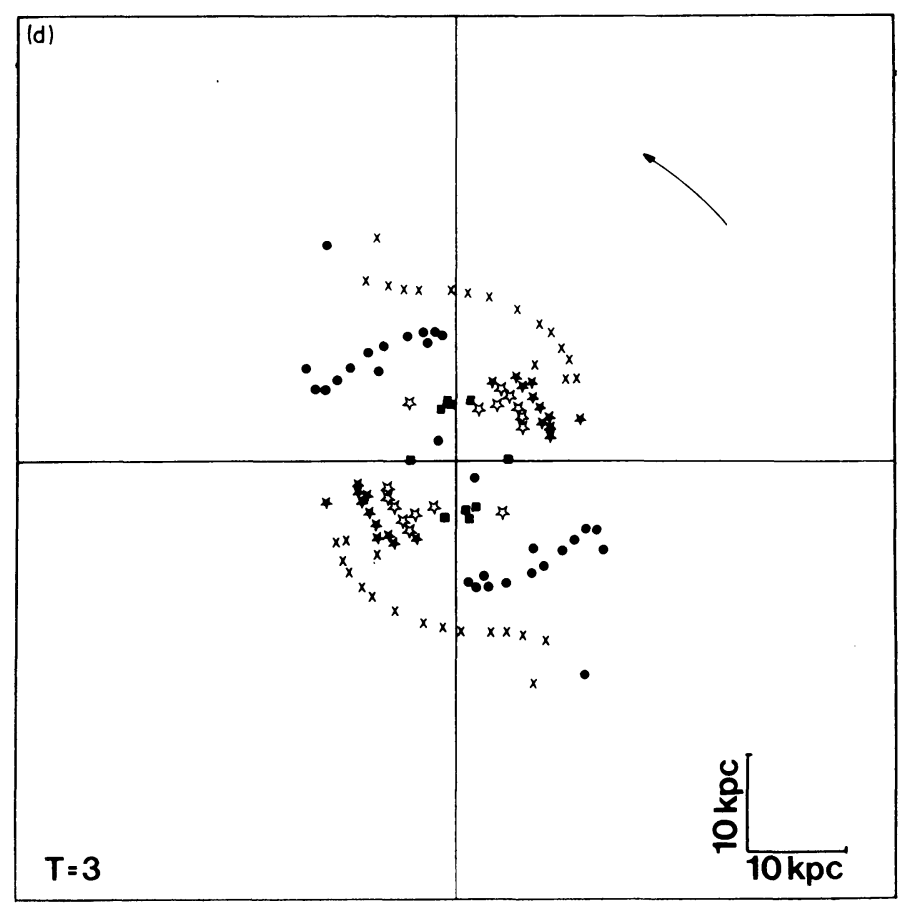

Fig. 2d. 


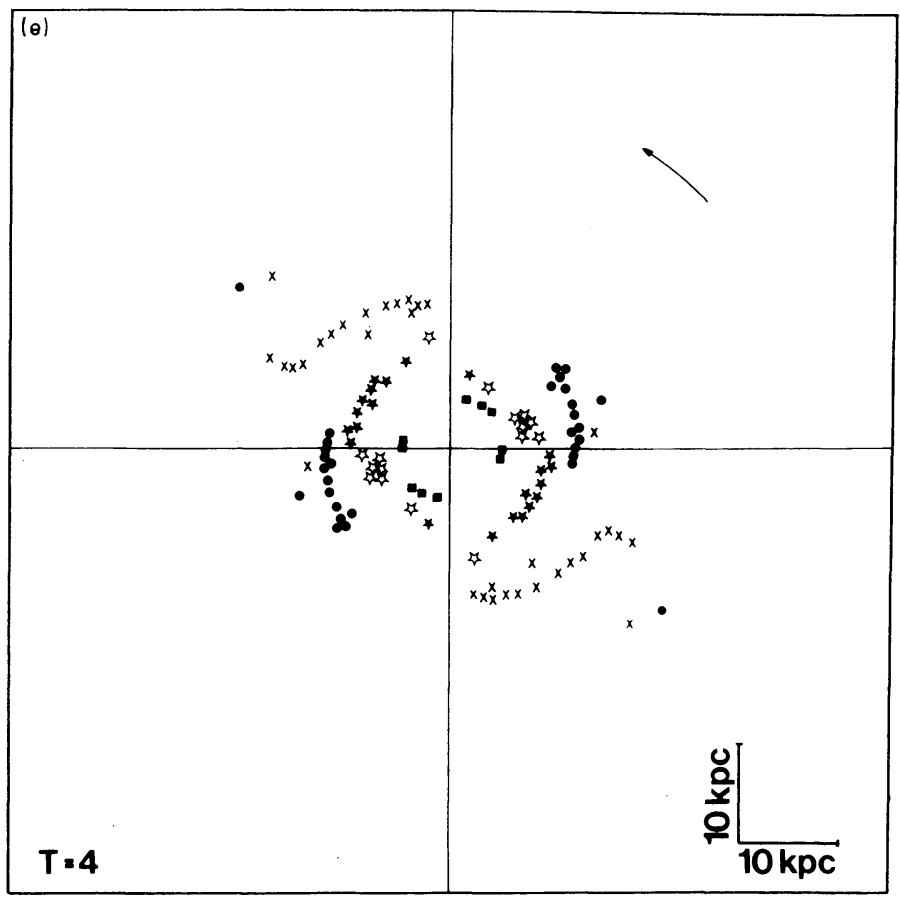

Fig. 2e.

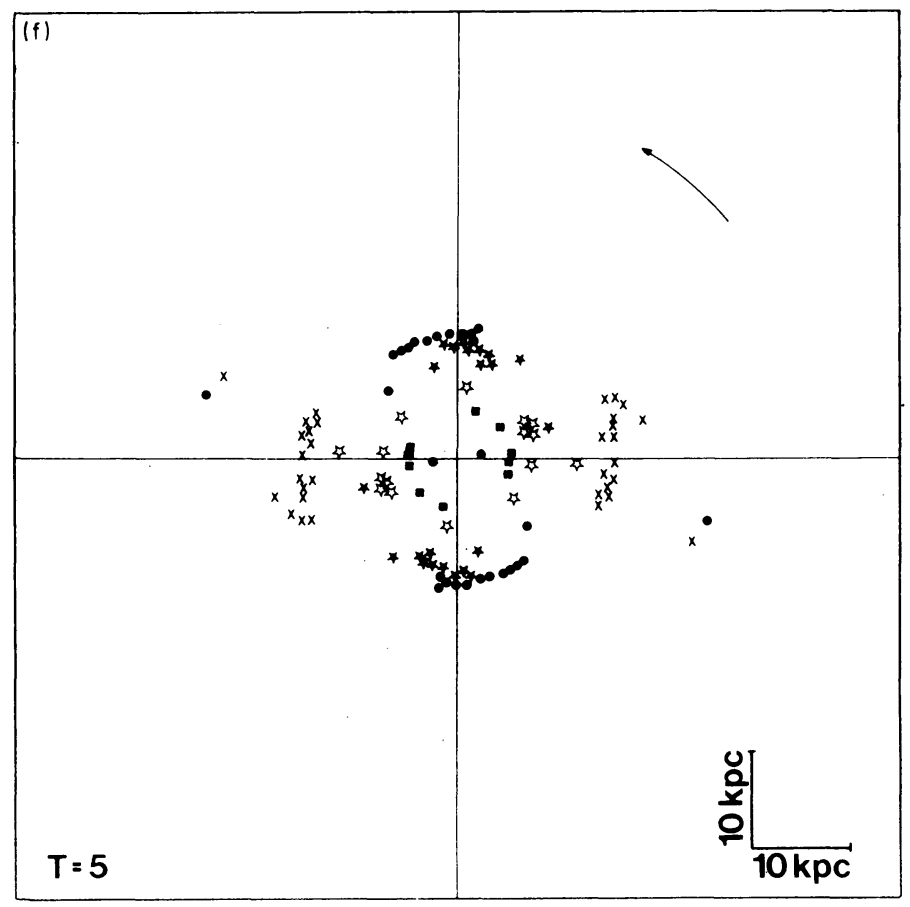

Fig. $2 \mathrm{f}$. 


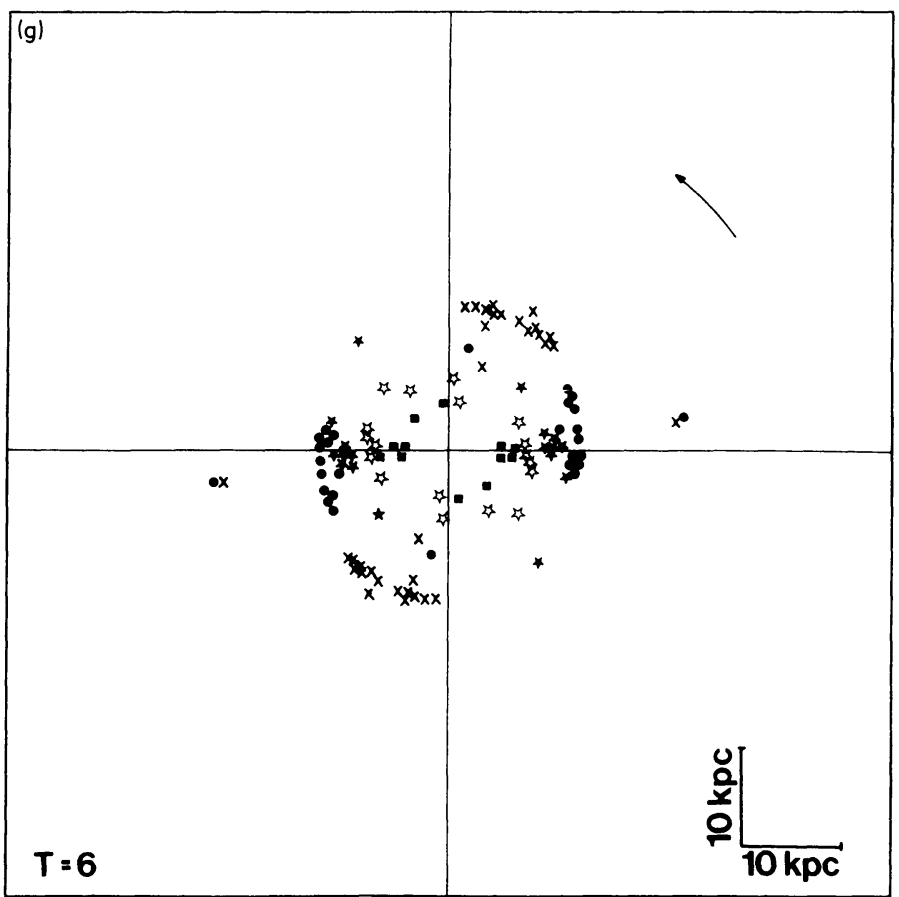

Fig. $2 \mathrm{~g}$.

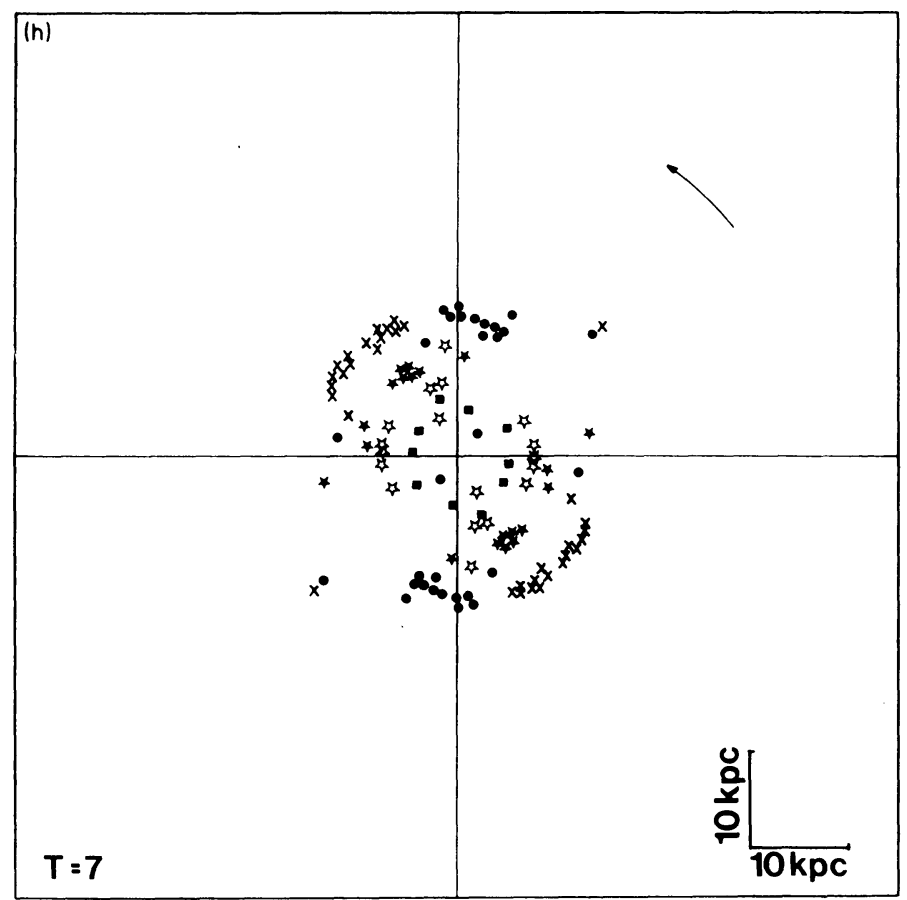

Fig. 2 (a) à (h). Deux fragments de même masse, $0.2 \times 10^{10} M_{\odot}$. sont éjectés dans deux directions opposées. On voit apparaître des bras, Figures (b) à (e) qui cèdent la place à une structure qui rappelle une spirale barrée, $(\mathrm{g})$ et $(\mathrm{h})$. 


\section{3. ÉJECTION SYMÉTRIQUE DE DEUX MASSES ÉGALES, CHACUNE, À $0.5 \times 10^{10} M_{\odot}$}

Les vitesses initiales sont de $2600 \mathrm{~km} \mathrm{~s}^{-1}$. Figure 3. Les étoiles ont été suivies pendant 10 unités de temps. Dès $T=1$ on voit apparaître un bras qui devance d'abord la rotation du disque puis se déforme et suit le disque pendant près de 4 unités de temps. Au delà de ce temps la structure du système devient confuse.

D'autres expériences numériques sont en cours, pour examiner notamment si l'on peut obtenir une structure spirale qui persiste plus longtemps.

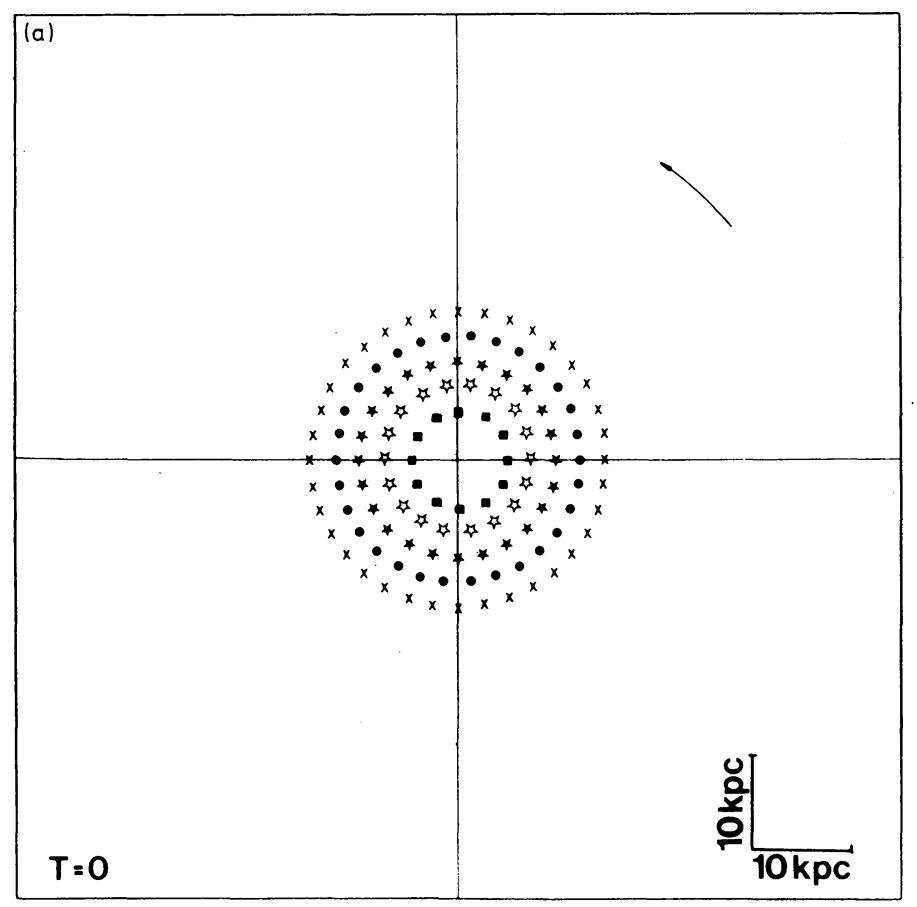

Fig. 3a. 


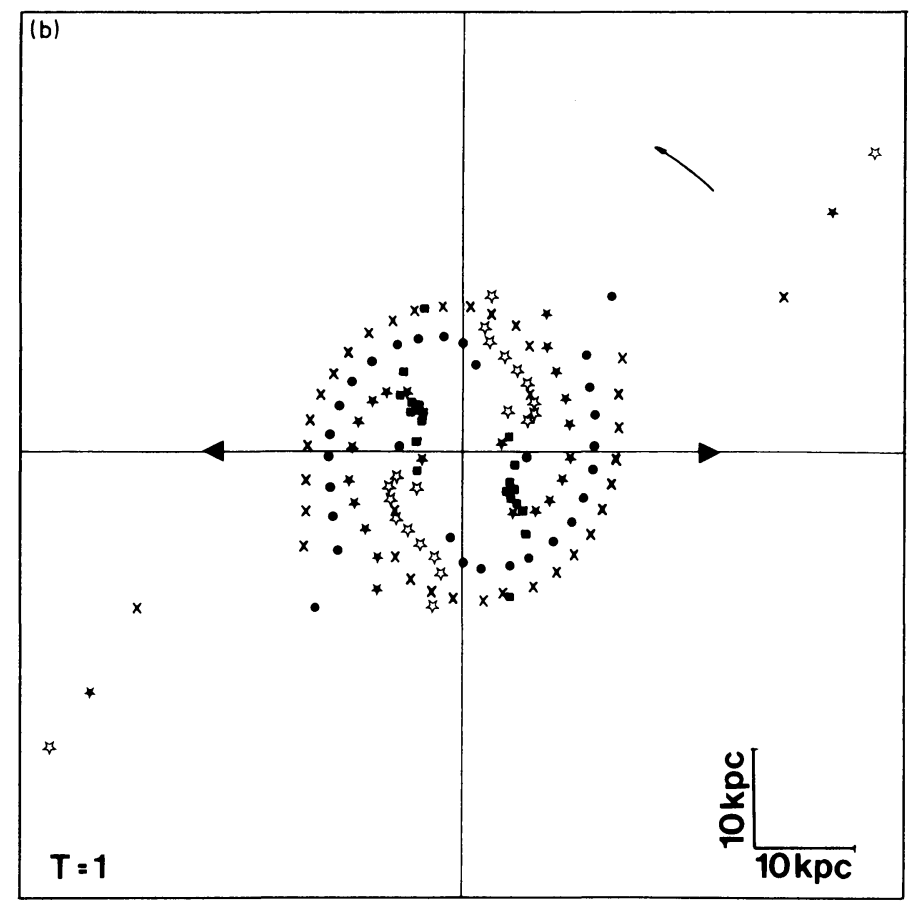

Fig. 3b.

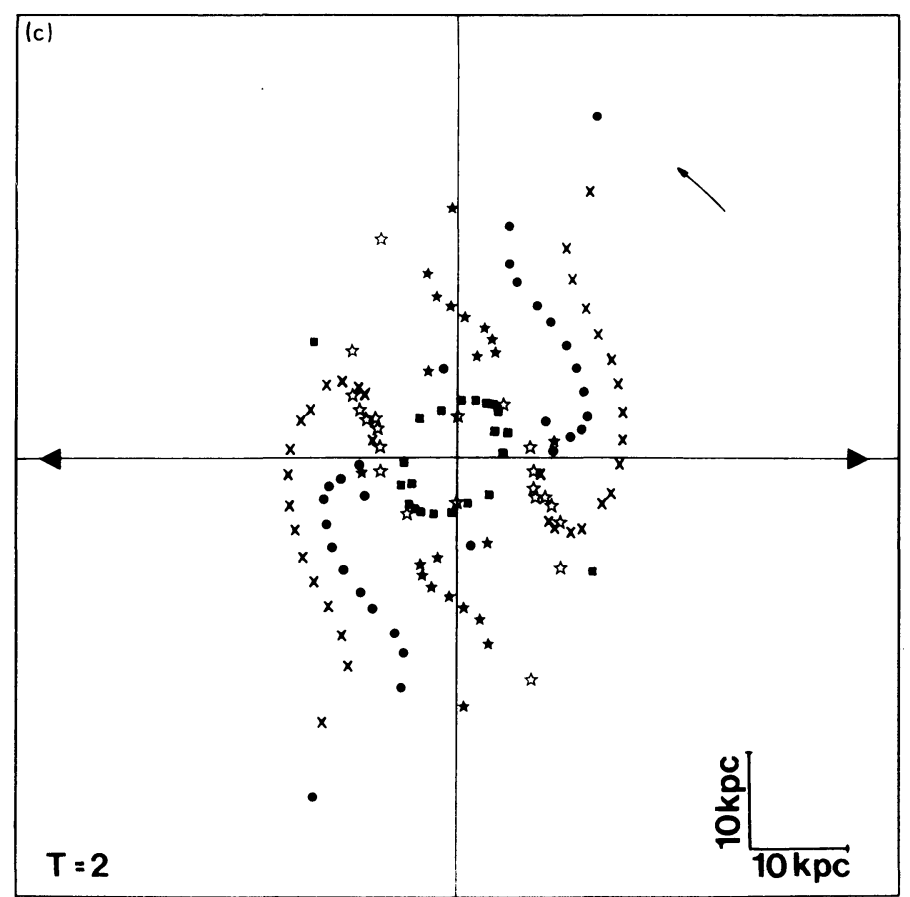

Fig. 3c. 


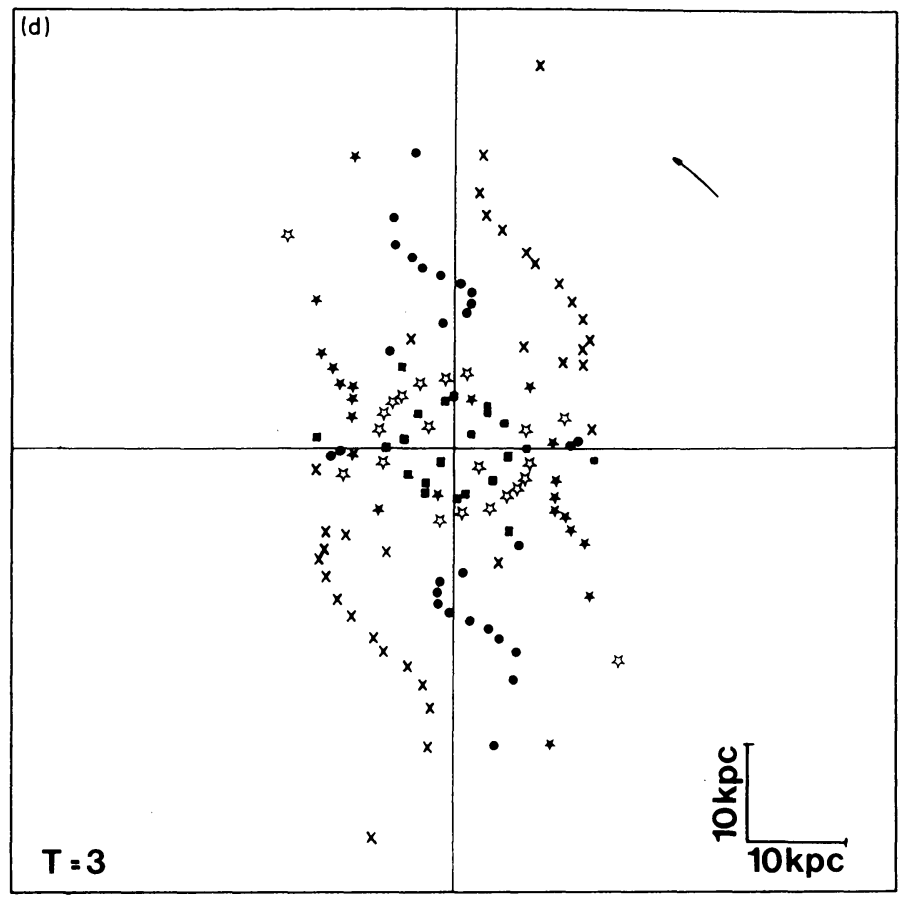

Fig. Bd.

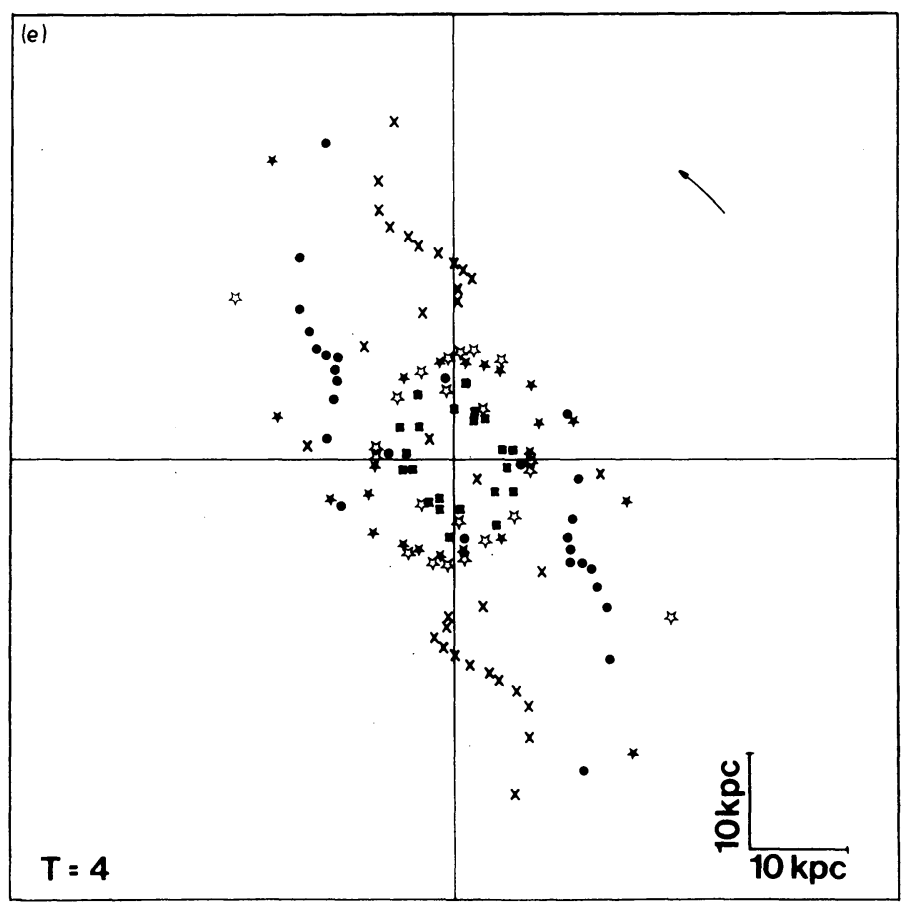

Fig. Be.

https://doi.org/10.1017/S0074180900015722 Published online by Cambridge University Press 


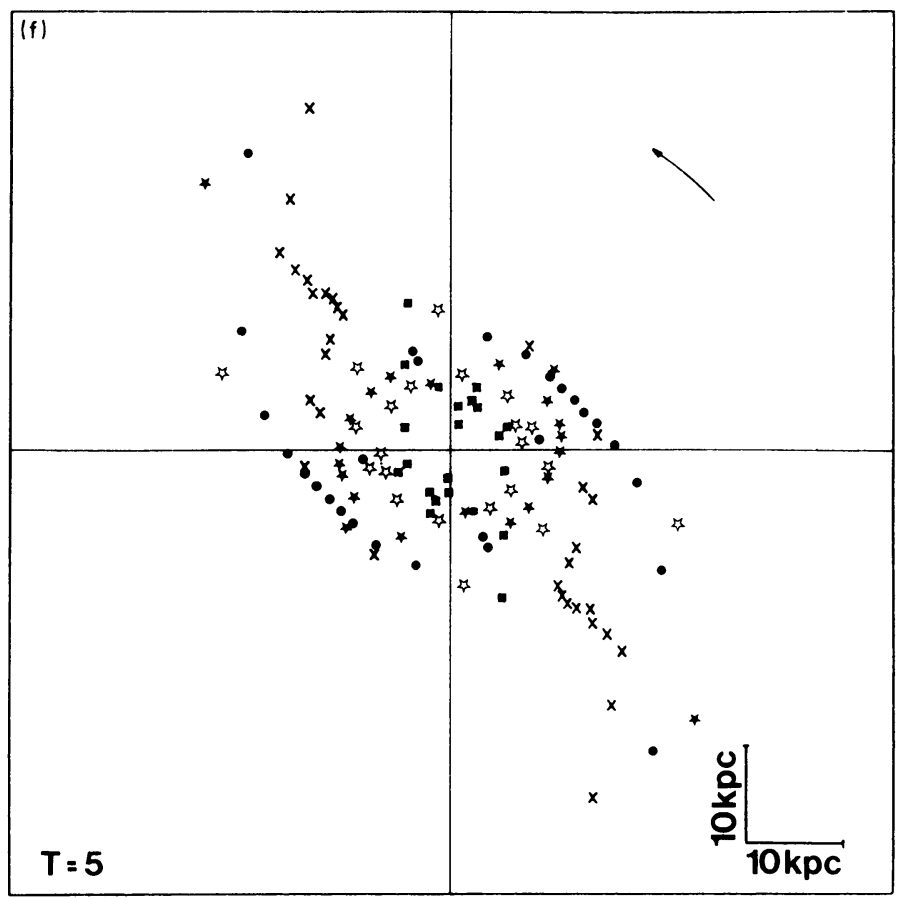

Fig. 3f.

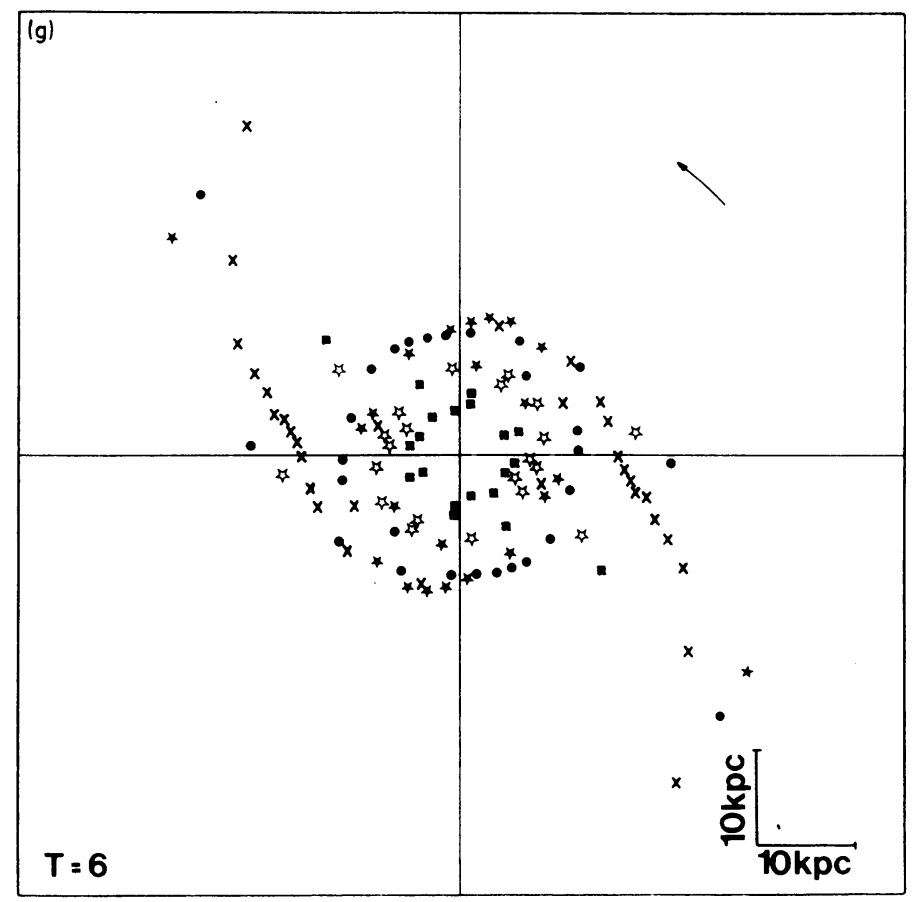

Fig. 3g. 


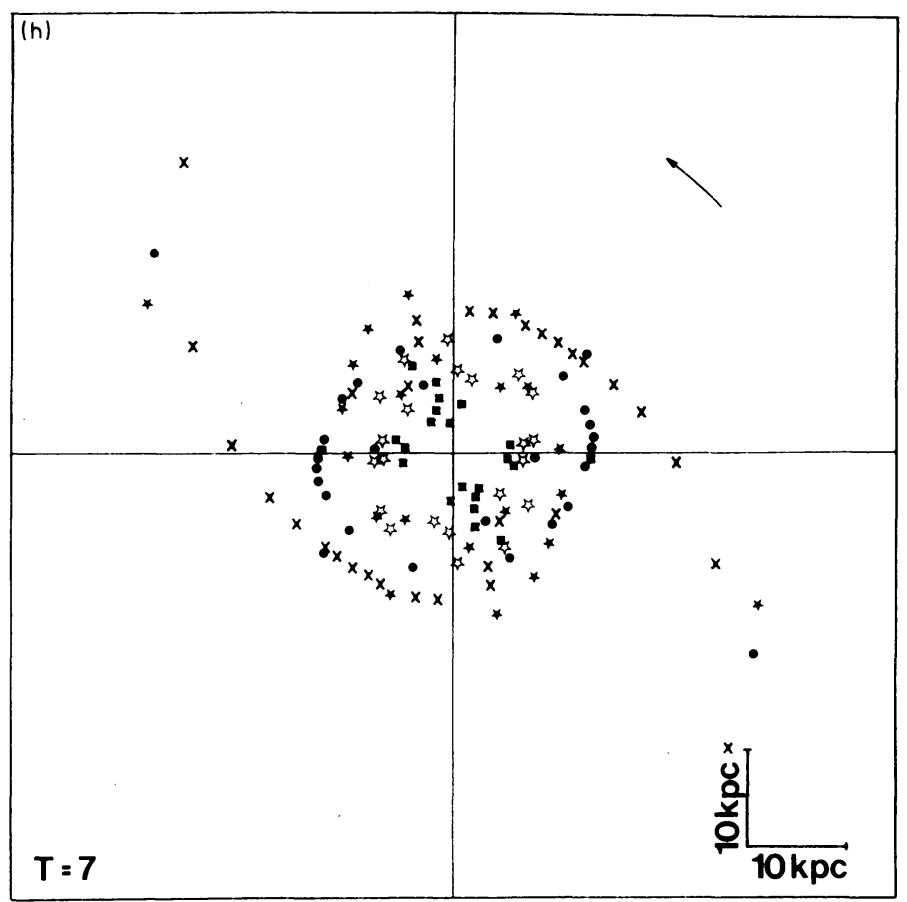

Fig. $3(\mathrm{a}) \mathrm{à} \mathrm{(h).} \mathrm{Deux} \mathrm{fragments} \mathrm{de} \mathrm{même} \mathrm{masse} 0.5 \times 10^{10} M_{\odot}$, sont éjectés dans deux directions opposées. Une structure de type spiral apparaît sur (b); elle est remplacée ensuite par des bras traînant, (g) et (h).

\section{Bibliographie}

Arp, H.: 1969, Astron. Astrophys. 3, 418.

O’Connell, D. J. K. (ed.): 1971, Nuclei of Galaxies, North-Holland Publ. Co., Amsterdam, London. Toomre, A. and Toomre, Y.: 1972, Astrophys. J. 178, 623.

Van der Kruit, P. C.: 1971, Astron. Astrophys. 15, 110.

\section{DISCUSSION}

Contopoulos: What is the life-time of the spiral feature?

Hayli: About $5 \times 10^{8} \mathrm{yr}$.

King: Why did you choose this particular ejection velocity?

Hayli: For the chosen velocities ejected bodies arrive at infinity with zero velocity. 